\title{
Effect of treadmill exercise training on spatial and temporal gait parameters in subjects with chronic stroke: A preliminary report
}

\author{
Shawnna L. Patterson, MD, PhD; ${ }^{1-2}$ Mary M. Rodgers, PhD, PT; ${ }^{3}$ Richard F. Macko, MD; ${ }^{1-2,4}$ Larry W. \\ Forrester, PhD $^{1,3-4 *}$ \\ ${ }^{1}$ Baltimore Department of Veterans Affairs (VAMC) Medical Center Geriatric Research, Education, and Clinical Cen- \\ ter, Baltimore, MD; ${ }^{2}$ Department of Neurology, Baltimore VAMC, Baltimore, MD; Departments of ${ }^{3}$ Physical Therapy \\ and Rehabilitation Science and ${ }^{4}$ Neurology, University of Maryland School of Medicine, Baltimore, MD
}

\begin{abstract}
The effects of task-repetitive locomotor training on stroke patients' spatial and temporal gait parameters during unassisted walking are not well understood. This study determined the effects of treadmill aerobic exercise (T-EX) on spatial and temporal gait parameters that underlie changes in overground walking function. Thirty-nine subjects with hemiparetic stroke underwent T-EX three times weekly for 6 months. We measured the subjects pre- and posttraining on 30-foot timed walks and 6-minute distance walks with usual assistive devices and on an $8 \mathrm{~m}$ instrumented walkway without assistive devices. T-EX improved 30 -foot walks by $17 \%$ and 6 -minute walks by $23 \%$. Unassisted walking velocity increased $22 \%$, stride length increased $13 \%$, and cadence increased $7 \%$. Paretic and nonparetic step lengths increased significantly, and respective step times decreased significantly. Interlimb symmetry did not change. This study presents preliminary evidence that changes in spatial and temporal gait parameters contribute to the increased velocity of subjects with stroke after T-EX.
\end{abstract}

Key words: cadence, exercise, function, gait, hemiparesis, rehabilitation, stride length, stroke, symmetry, treadmill, velocity.

\section{INTRODUCTION}

Of the 750,000 strokes occurring annually in the United States, 50 percent lead to chronic movement disabilities due to residual hemiparesis in the limbs [1-2]. Among subjects with such impairments, restoration of walking function is one of the primary targets in post- stroke rehabilitation, given the importance of mobility in the performance of activities of daily living, maintenance of independence, and reduction of other health problems associated with immobility and sedentary lifestyle [3]. Prior studies have shown that gait velocities may decrease [4] and gait parameters remain unchanged [5] over the course of natural recovery. Within the limited time frame for conventional poststroke rehabilitation [6], the degree of therapeutic focus and intensity may vary for gait retraining [7-8]. Also, the amount of time spent performing aerobic exercise in a typical poststroke rehabilitation therapy session has been reported to be only a few minutes [9]. Together, these facts suggest insufficient task repetition for motor learning.

Recent studies have investigated the longer term effects of sustained task-repetitive gait training among chronic stroke survivors who were presumed to have plateaued in gait recovery by 6 months poststroke. For

\footnotetext{
Abbreviations: $\mathrm{AFO}=$ ankle-foot orthosis, $\mathrm{CNS}=$ central nervous system, HRR = heart rate reserve, $\mathrm{SD}=$ standard deviation, T-EX = treadmill aerobic exercise, VA = Department of Veterans Affairs, $\mathrm{VO}_{2}=$ oxygen consumption.

*Address all correspondence to Larry W. Forrester, PhD; Department of Physical Therapy and Rehabilitation Science, University of Maryland School of Medicine, 100 Penn Street, Suite 115, Baltimore, MD 21201-1082; 410-706-5212; fax: 410-706-6387. Email: Iforrester@som.umaryland.edu DOI: 10.1682/JRRD.2007.02.0024
} 
example, Hesse et al. showed that treadmill training with partial body-weight support improved gait velocity and related cadence and stride-length parameters in subjects with subacute and chronic stroke [10]. In another study, subjects with chronic stroke either using an electromechanical gait trainer with or without functional electrical stimulation or participating in overground walking therapy increased their gait velocity in timed floor walks [11]. Another recent study of subjects with chronic hemiparesis reported that a 12-week program of partial body weight-supported treadmill training coupled with overground walking practice led to faster floor walking velocities with increased step lengths and cadence in most of the subjects [12]. In each of these examples, the focus was on augmentation of the walking exercise with guided placement of the paretic leg either by therapists or a machine. Additionally, we found in a randomized controlled study that a 6-month progressive treadmill aerobic exercise (T-EX) training program without emphasis on gait patterning not only improved cardiovascular fitness but also increased gait velocity in subjects who averaged more than 3 years poststroke [13]. Even at 3 months, a small subset of these subjects showed increased overground velocity, cadence, and symmetry with decreased stance and swing times but no changes in stride length [14]. Taken together, these examples indicate that many stroke survivors can improve ambulatory function beyond the usual recovery period if they receive intensive walking practice that may or may not be complemented by augmenting stepping patterns.

A possible mechanism for the functional gains from T-EX after stroke is its potential to stimulate reorganization in the central nervous system (CNS) and thereby improve lower-limb motor control and gait patterning. In support of this possibility, temporal and weight-bearing symmetry have been shown to immediately improve when subjects with hemiparetic gait walk on a treadmill compared with when they walk overground at the same velocity [15]. Also, these changes are accompanied by shifts in timing of lower-limb electromyography activation [16]. Importantly, these findings indicate that the alteration of the gait pattern is due not just to the mechanical effects of the treadmill moving the legs but also to the responsiveness of the neuromotor system. While the short-term responsiveness to the treadmill stimulus alters temporal gait parameters, we do not know whether longterm T-EX training without explicit practice of gait patterning via therapist assistance (or mechanical guidance) will induce improvements that carry over to unassisted overground walking patterns.

Increased gait velocity, not improved gait patterning, is often the outcome measure for evaluating gait function. Gait velocity is determined by cadence and stride length, and a given velocity can be achieved through different combinations of cadence and stride length. This study determined whether global increases in cardiovascular fitness and walking velocity are also accompanied by significant alterations in spatial, temporal, and symmetry measures of the unassisted overground gait patterns in subjects with chronic stroke. Our previous studies suggest that T-EX may alter temporal symmetry and that velocity gains may be due more to increased cadence than to longer stride length [15-16]. Thus, we hypothesized that 6 months of T-EX would increase subjects' unassisted overground walking velocity as a result of faster cadence rather than increased stride length. Additionally, we hypothesized that 6 months of T-EX would improve subjects' interlimb symmetry variables, as defined by paretic to nonparetic ratios of selected gait cycle parameters.

\section{METHODS}

\section{Participants}

Forty-two subjects completed 6 months of T-EX and were tested pre- and posttraining on 30-foot walks, 6-minute walks, unassisted floor walks, and peak oxygen consumption $\left(\mathrm{VO}_{2}\right)$ measures. Three subjects were excluded secondary to outlier analysis (30-foot walk velocity $>2$ standard deviation [SD] slower than the overall group mean). A subset of participants was included in a previously published report [13]. Demographic details and deficit characteristics of the 39 subjects used in the final analyses are presented in Table 1.

Subjects were older than 40 years of age and had chronic ( $>6$ months) stroke with residual hemiparetic gait. Subjects with severe or active renal, cardiac, pulmonary, or hematological illness were excluded from enrolling. Subjects were recruited from the Baltimore Department of Veterans Affairs (VA) Maryland Health Care System and the University of Maryland Medical Center. The study was approved by the University of Maryland Institutional Review Board and the Baltimore VA Research and Development Committee. All subjects provided written informed consent. 
Table 1.

Demographics of subjects with chronic stroke $(n=39)$.

\begin{tabular}{lc}
\hline \multicolumn{1}{c}{ Demographic } & Mean \pm SD (Range) or \% \\
\hline Age (yr) & $64 \pm 8(47-78)$ \\
Sex: Male & 64 \\
Race/Ethnicity & 41 \\
White & 51 \\
$\quad$ African American & $28.6 \pm 6.1(20.3-45.2)$ \\
Body Mass Index & \\
Latency Since Stroke (mo) & 62 \\
Paretic Side & 38 \\
Right & 51 \\
Left & \\
Ankle-Foot Orthosis & 31 \\
Assistive Device & 41 \\
None & 18 \\
Single Point Cane & 10 \\
Quad Cane & \\
Walker & \\
SD = standard deviation. & \\
\hline \hline
\end{tabular}

\section{Intervention}

Subjects participated in T-EX three times a week for 6 months [13]. Training started with 10 to 20 minutes at low intensity (40\% to $50 \%$ heart rate reserve [HRR]) [17]. The duration was progressed 5 minutes every 2 weeks and the intensity was progressed 5 percent HRR every 2 weeks, with a goal of 40 minutes at 60 to 70 percent HRR.

\section{Measures}

Body mass index was determined from the subject's mass and height during a baseline medical evaluation. Use of an ankle-foot orthosis (AFO) or assistive device (none, single point cane, quad cane, or walker) was documented. Cardiovascular fitness, overground walking function, and spatiotemporal gait parameters were evaluated at baseline and again after 6 months of T-EX [13].

Overground walking function with usual assistive devices was evaluated by the 30 -foot timed walk and the 6-minute walk for distance. For the 30-foot walk [18], subjects were asked to walk the specified distance at their self-selected, most comfortable walking speed. We recorded both the time and number of steps required to walk $30 \mathrm{ft}(9.1 \mathrm{~m})$ to estimate average velocity and step cadence. This test was repeated three times, with a short rest period between each trial, and the average of the three trials was used in the data analysis. For the 6-minute walk [19], subjects were instructed to cover as much distance as possible during 6 minutes while walking around a $30.5 \mathrm{~m}$ course marked with orange cones in a straight hospital corridor. For both floor walking tests, subjects wore a gait belt for safety and were closely monitored by a member of the research staff.

To further define eligibility for T-EX training and exercise prescription intensity, we had subjects also undergo a treadmill tolerance test and a treadmill exercise stress test [20]. Those able to walk for at least 3 minutes at $\geq 0.09 \mathrm{~m} / \mathrm{s}$ during the treadmill exercise stress test returned for a peak treadmill exercise test with open-circuit spirometry to measure cardiovascular fitness (peak $\mathrm{VO}_{2}$ ) [21-22]. Treadmill tests were terminated for angina, arrhythmia, decreasing blood pressure, or fatigue as recommended by American College of Sports Medicine criteria or for overt signs of gait instability [17].

On a separate day, subjects performed a series of overground walks without their usual assistive device while traversing a $7.3 \mathrm{~m}$ instrumented walkway (GAITRite ${ }^{\mathrm{TM}}$, CIR Systems; Clifton, New Jersey). Again, they wore a gait belt and were attended by research staff as they walked at their self-selected speed. Three trials were collected, with 2-minute seated rests between trials. All but the most impaired subjects were allowed $2 \mathrm{~m}$ before and after the active surface of the GAITRite to accelerate and decelerate. Where necessary, steps taken during initial acceleration and final deceleration were removed during postprocessing to ensure that subjects' best steady state walking patterns determined the gait parameters.

Outcomes from the GAITRite data processing software included global measures of average velocity, cadence, and stride length. Elemental gait parameters were determined for the paretic and nonparetic limbs separately, including individual step lengths, step times, and the absolute and relative times of stance and swing phases. Measures of interlimb symmetry were defined as ratios of paretic to nonparetic values of the spatial and temporal variables. Relative temporal phasing was also calculated as the time within the nonparetic gait cycle (\%) at which the paretic foot touched down. Relative distance phasing was calculated as the percentage of the nonparetic stride length accounted for by the paretic step length. The mean values for each variable from each trial were averaged across the three trials to calculate preferred speed walking patterns.

\section{Data Analysis}

Using SPSS 13.0 (SPSS Inc; Chicago, Illinois), we analyzed paired $t$-tests to evaluate differences between pre- and posttraining evaluations and Pearson correlation 
coefficients to compare laboratory and clinical velocity measures. Paired $t$-tests were done sequentially, reflecting a hierarchical approach from global measures (e.g., velocity) to specific measures within temporal and spatial domains. If significance was found, pre- and posttraining component-specific measures were compared; when significance was not found, the analysis was concluded. The significance level was set at $p<0.05$.

\section{RESULTS}

The subjects' demographics and use of an AFO or assistive device are listed in Table 1. At the completion of 6 months of T-EX, the subjects' mean \pm SD training duration was $34.7 \pm 7.4$ minutes (range $=20-50$ minutes). Improvements were seen in 30-foot walk velocity, 6-minute walk distance, and cardiovascular fitness after completing T-EX (Table 2). Measures of unassisted overground walking also improved (Table 2). Mean values for velocity, stride length, and cadence increased significantly. Paretic and nonparetic step lengths increased significantly, while respective step times decreased significantly. Significant decreases were seen in relative paretic stance time and increases in relative paretic swing time. Similar changes were seen for the nonparetic limb, although they were nonsignificant. Additionally, relative double support time decreased. Measures of interlimb symmetry did not significantly change, as shown in Table 3.

Self-selected gait velocity with a usual assistive device tended to be greater than overground walking without an assistive device, but they were significantly correlated ( $r=0.848, p<0.001)$ at baseline and more strongly correlated after 6 months of treadmill training ( $r=0.917, p<0.001)$. Overground assisted gait velocity and unassisted gait velocity correlated highly to peak treadmill walking velocity sustained during a graded treadmill exercise test. The correlations were robust at baseline ( $r=0.800, p<0.001$ and $r=0.788, p<0.001$, respectively) and at 6 months ( $r=0.843, p<0.001$ and $r=0.815, p<0.001$, respectively). Percent change in

Table 2.

Pre- and posttraining functional and unassisted gait parameters for subjects with chronic stroke $(n=39)$.

\begin{tabular}{|c|c|c|c|}
\hline \multirow{2}{*}{ Parameter } & \multicolumn{2}{|c|}{ Mean \pm Standard Deviation } & \multirow{2}{*}{ Mean \% Change } \\
\hline & Baseline & 6 Months & \\
\hline \multicolumn{4}{|l|}{ Functional } \\
\hline 30-Foot Walk Velocity (m/s) & $0.64 \pm 0.29$ & $0.74 \pm 0.29^{*}$ & 16.6 \\
\hline Peak Oxygen Consumption (mL/kg/min) & $14.4 \pm 4.6$ & $17.3 \pm 5.6^{\dagger}$ & 22.4 \\
\hline \multicolumn{4}{|l|}{ Global Gait } \\
\hline Cadence (steps/min) $)^{\S}$ & $81.0 \pm 16.3$ & $86.0 \pm 16.4^{*}$ & 7.0 \\
\hline \multicolumn{4}{|l|}{ Spatial Gait } \\
\hline Paretic Step Length (cm) & $41.7 \pm 13.0$ & $46.5 \pm 12.9^{\dagger}$ & 15.4 \\
\hline Nonparetic Step Length (cm) & $36.4 \pm 16.6$ & $40.1 \pm 18.1^{*}$ & 12.5 \\
\hline \multicolumn{4}{|l|}{ Temporal Gait } \\
\hline Nonparetic Stance (\% cycle) & $76.2 \pm 7.6$ & $75.1 \pm 7.9$ & -1.3 \\
\hline Double Support (\% cycle) & $42.4 \pm 11.5$ & $39.5 \pm 11.9$ & -5.8 \\
\hline Paretic Swing (\% cycle) & $33.8 \pm 5.0$ & $35.7 \pm 5.3^{\mathbb{q}}$ & 6.6 \\
\hline Nonparetic Swing (\% cycle) & $23.8 \pm 7.6$ & $24.9 \pm 7.9$ & 5.8 \\
\hline \multicolumn{4}{|c|}{$\begin{array}{l}{ }^{*} p<0.005 . \\
{ }^{\dagger} p<0.001 . \\
{ }^{\dagger} \text { Healthy older adults: } 110-150 \mathrm{~cm} / \mathrm{s} \text {. Maki B. Gait changes in older adults: Predictors of falls or indicators of fear. J Am Geriatr Soc. 1997;45(3):313-20. [PMID: 9063277 } \\
{ }^{\S} \text { Healthy older adults: } 90-125 \text { steps/min. Ibid. } \\
{ }^{{ }^{\dagger}} p<0.05 \text {. }\end{array}$} \\
\hline
\end{tabular}


PATTERSON et al. Treadmill training and gait parameters in stroke

Table 3.

Pre- and posttraining interlimb symmetry variables (ratio of paretic to nonparetic limb) for subjects with chronic stroke $(n=39)$.

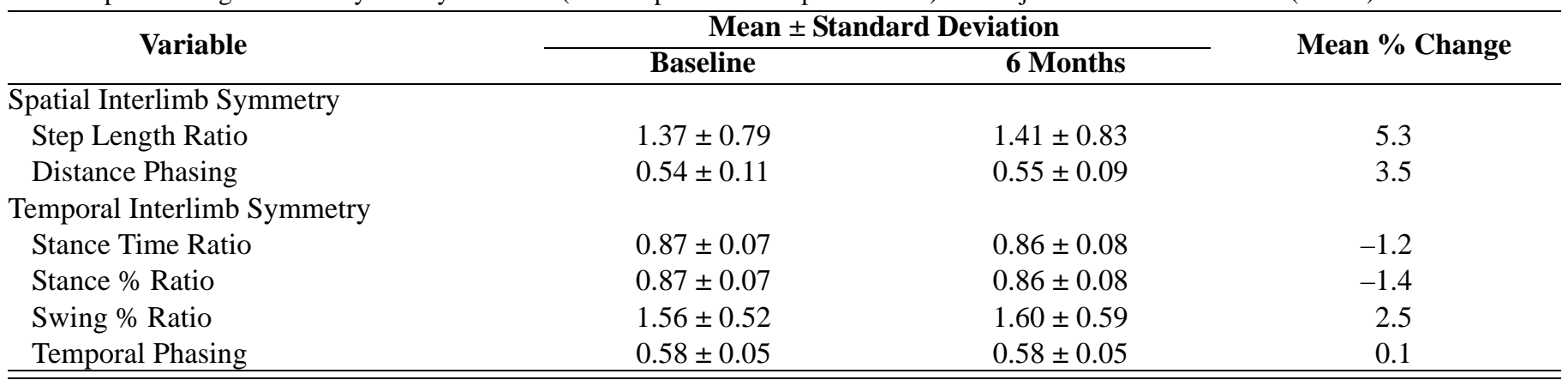

peak $\mathrm{VO}_{2}$ correlated to percent change in testing treadmill speed $(r=0.433, p<0.01)$ and percent change in training speed $(r=0.422, p<0.01)$ but not to overground walking speed with an assistive device $(r=0.130, p=$ $0.46)$ or without an assistive device $(r=0.049, p=0.77)$.

\section{DISCUSSION}

Progressive T-EX induces improvements in overground walking function, as indexed by increased walking velocity. Increased walking velocity occurred during multiple tasks, including assisted overground walking, unassisted overground walking, 6-minute walk distance, treadmill training, and treadmill testing. Analysis of spatial and temporal gait parameters in unassisted overground walking showed that velocity increased because of increased stride length and cadence. Symmetry variables in unassisted overground walking, however, did not change. Overall, these results suggest that progressive T-EX produces locomotor training that carries over to a variety of functional conditions.

This study not only demonstrates that 6 months of an aerobically based treadmill training program improves global indexes of gait function in chronic stroke but also begins to assess elemental spatial and temporal contributions to increased gait velocity. It differs from shorter duration interventions that use a treadmill with partial body-weight support $[10,13]$ or a motorized gait trainer [11] because it emphasizes the level of aerobic intensity and the massed practice accrued over 6 months. We do not know whether similar gains could be made with less training, although our previous work suggests at least some effects are realized within 3 months [14], a duration that Plummer et al. also found effective [12]. Previous comparisons between 3-month and 6-month outcomes showed continued gains across the entire period [13].

The gait parameters obtained in this study are similar to those published in cross-sectional studies reporting gait characteristics of subjects with poststroke hemiparesis [23-24]. Stride length accounted for nearly two-thirds, and cadence one-third, of the increased velocity seen in this study. Increased stride length is due to increases in bilateral step lengths. Both paretic and nonparetic step lengths demonstrated a clinically significant gain of nearly $5 \mathrm{~cm}$. These spatial improvements, seen after longterm T-EX, may be more beneficial than the hypothesized improvements in the temporal variable, cadence. The increased step lengths in both legs suggest improved paretic leg function. Changes in the paretic leg may allow increased propulsion, which leads to increased nonparetic step length [25]. Conversely, increased paretic step length may be due to an increased range of motion in the paretic leg joints that allows the limb to swing farther forward. Further biomechanical studies are needed to evaluate the underlying mechanism.

In addition to stride length, cadence increased. Increased cadence is due to decreases in bilateral step times. Significant decreases were seen in relative paretic stance time, relative paretic swing time, and relative double support time. Decreased double support time may be considered a surrogate indicator of improved balance control. Older adults walk more slowly with increased double support time [26]. In general, decreased gait velocity predicts disability in healthy older adults [27]. Increased double support time may be related to an increased risk of falls, although one study suggests it may be more related to a fear of falling [28]. Another study of walking therapy in stroke has shown increased gait velocity and improved balance [11]. Our results suggest 
that T-EX can decrease double support time and increase single stance time and velocity. These changes in patterning may reflect improved balance, although what proportion is due to increased velocity is unclear.

Increased assisted and unassisted overground walking velocities suggest that gait changes apparent during treadmill training are carried over to overground walking and that T-EX training improves gait under multiple task environments. Our prior studies demonstrated improved symmetry secondary to immediate short-term adaptations in untrained subjects with stroke on the treadmill [15-16]. In comparison, the current results suggest that while gait velocity improves in multiple environments, improvements in symmetry are not transferred to independent overground walking. This lack of transfer could be due to the individual's continued reliance on an assistive device when off the treadmill, task repetition reinforcement of asymmetry on the treadmill, absence of practice of unassisted walking, and a reduced balance challenge secondary to handrail use during training. The global increase in gait velocity without improvements in symmetry suggests that compensatory strategies are retained and amplified. Introduction of additional training variables such as increased duration and periodic balance challenges by limiting handrail support may provide a more effective stimulus for altering symmetry.

The high correlation between assisted and unassisted overground gait velocity allows comparison of the spatiotemporal gait parameters with the clinical index of gait velocity that evaluates disability in subjects with stroke. We previously reported that increased training velocity predicted improved cardiovascular fitness and that increased training duration predicted improved 6-minute walk distance [13]. The results of this analysis again support the previous finding that changes in training velocity correlate with changes in cardiovascular fitness; however, an uncoupling of the relationship between training duration and 6-minute walk distance exists. Additionally, changes in assisted and unassisted overground walking velocity are not correlated to changes in cardiovascular fitness. This finding is counterintuitive and suggests that we must consider factors other than training velocity and duration in our task-repetitive model of rehabilitation.

Along these lines, we note that the present 6-month program is much longer than other interventions that focus more on gait patterning than on aerobic intensity. The effects on gait parameters may be realized much more rapidly when the focus of massed practice is spe- cific to those outcomes [10-12]. We do not know whether similar gains would be made if the T-EX program were shortened, although our previous work suggests that some effects may be realized within 3 months [14], a duration also found to be effective by Plummer et al. [12]. While explicit comparisons of outcomes among the various approaches to gait rehabilitation are confounded by methodological differences, the common theme is that goal-directed locomotor practice can lead to improved function through motor learning.

With respect to motor learning and the absence of symmetry changes, specificity of practice for symmetry was not an overt focus of the T-EX paradigm, although some degree of symmetry was imposed by the treadmill. Thus, the T-EX stimulus plausibly evoked some degree of motor learning through repetition of more symmetrical stepping over 6 months of practice. Preliminary functional magnetic resonance imaging in a subset of these subjects has been reported for paretic knee movement [29]. These data suggest that new CNS activation, and perhaps reorganization, occurs in the bilateral subcortex after 6 months of T-EX, providing initial evidence of brain plasticity secondary to task-repetitive locomotor training. How CNS reorganization interacts with increased walking velocity, stride length, and cadence (but not interlimb symmetry) is not well understood and requires further study.

Limitations of this study include the large range of the subjects' timed and distance walks, the absence of a control group, and limited details about gait biomechanics. Further studies with a control group are needed to evaluate the specificity of the effect of T-EX and the effects of T-EX on angular kinematics and torques of the lowerlimb joints. These evaluations will help us determine whether the increased velocity is secondary to changes across the different joints in each limb and evaluate the emergence of other compensatory strategies.

\section{CONCLUSIONS}

T-EX improves gait velocity secondary to increased stride length, and to a lesser extent, cadence. This increased velocity is carried over to multiple functionally relevant conditions. Additionally, T-EX improves indexes of dynamic stability without changing symmetry. Further studies are needed to optimize the translation of treadmill gait training to overground walking. Additionally, specific training for symmetry may have a stronger effect on 
improvements in spatiotemporal gait parameters, such as cadence or step length, thereby creating more efficient gait.

\section{ACKNOWLEDGMENTS}

We would like to thank John D. Sorkin, MD, PhD, for statistical consultation.

This material was based on work supported by the VA Medical Center Baltimore Geriatric Research, Education, and Clinical Center; VA Medical Center Rehabilitation Research and Development Center of Excellence in Exercise and Robotics Rehabilitation (grant B3688R); VA Rehabilitation Research and Development Career Development Award (grant B2375V); VA Research Enhancement Award Program in Stroke; and National Institute on Aging Claude D. Pepper Older Americans Independence Center (grant P60AG12583).

The authors have declared that no competing interests exist.

\section{REFERENCES}

1. Gresham GE, Phillips TF, Wolf PA, McNamara PM, Kannel WB, Dawber TR. Epidemiologic profile of long-term stroke disability: The Framingham study. Arch Phys Med Rehabil. 1979;60(11):487-91. [PMID: 508073]

2. LeBrasseur NK, Sayers SP, Ouellette MM, Fielding RA. Muscle impairments and behavioral factors mediate functional limitations and disability following stroke. Phys Ther. 2006;86(10):1342-50. [PMID: 17012638]

3. Reding M, Potes E. Rehabilitation outcome following initial unilateral hemispheric stroke. Life table analysis approach. Stroke. 1988;19(11):1354-58. [PMID: 3188120$]$

4. Wade DT, Collen FM, Robb GF, Warlow CP. Physiotherapy intervention late after stroke and mobility. BMJ. 1992; 304(6827):609-13. [PMID: 1559090]

5. Von Schroeder H, Coutts R, Lyden PD, Billings E Jr, Nickel VL. Gait parameters following stroke: A practical assessment. J Rehabil Res Dev. 1995;32(1):25-32. [PMID: 7760264]

6. Shah S, Vanclay F, Cooper B. Efficiency, effectiveness, and duration of stroke rehabilitation. Stroke. 1999;21(2):241-46. [PMID: 2305399]

7. Jette DU, Latham NK, Smout RJ, Gassaway J, Slavin MD, Horn SD. Physical therapy interventions for patients with stroke in inpatient rehabilitation facilities. Phys Ther. 2005; 85(3):238-48. [PMID: 15733048$]$
8. Latham NK, Jette DU, Slavin M, Richards LG, Procino A, Smout RJ, Horn SD. Physical therapy during stroke rehabilitation for people with different walking abilities. Arch Phys Med Rehabil. 2005;86(12 Suppl 2):S41-50. [PMID: 16373139]

9. MacKay-Lyons M, Makrides L. Cardiovascular stress during a contemporary stroke rehabilitation program: Is the intensity adequate to induce a training effect? Arch Phys Med Rehabil. 2002;83(10):1378-83. [PMID: 12370872]

10. Hesse S, Bertelt C, Schaffrin A, Malezic M, Mauritz KH. Restoration of gait in nonambulatory hemiparetic patients by treadmill training with partial body-weight support. Arch Phys Med Rehabil. 1994;75(10):1087-93.

[PMID: 7944913]

11. Peurala SH, Tarkka IM, Pitkänen K, Sivenius J. The effectiveness of body weight-supported gait training and floor walking in patients with chronic stroke. Arch Phys Med Rehabil. 2005;86(8):1557-64. [PMID: 16084808]

12. Plummer P, Behrman AL, Duncan PW, Spigel P, Saracino D, Martin J, Fox E, Thigpen M, Kautz SA. Effects of stroke severity and training duration on locomotor recovery after stroke: A pilot study. Neurorehabil Neural Repair. 2007; 21(2):137-51. [PMID: 17312089]

13. Macko RF, Ivey FM, Forrester LW, Hanley D, Sorkin JD, Katzel LI, Silver KH, Goldberg AP. Treadmill exercise rehabilitation improves ambulatory function and cardiovascular fitness in patients with chronic stroke: A randomized, controlled trial. Stroke. 2005;36(10):2206-11.

[PMID: 16151035]

14. Silver KH, Macko RF, Forrester LW, Goldberg AP, Smith GV. Effects of aerobic treadmill training on gait velocity, cadence, and gait symmetry in chronic hemiparetic stroke. Neurorehabil Neural Repair. 2000;14(1):65-71.

[PMID: 11228951]

15. Harris-Love ML, Forrester LW, Macko RF, Silver KH, Smith GV. Hemiparetic gait parameters in overground versus treadmill walking. Neurorehabil Neural Repair. 2001; 15(2):105-12. [PMID: 11811252$]$

16. Harris-Love ML, Macko RF, Whitall J, Forrester LW. Improved hemiparetic muscle activation in treadmill versus overground walking. Neurorehabil Neural Repair. 2004;18(3): 154-60. [PMID: 15375275]

17. American College of Sports Medicine. ACSM's guidelines for exercise testing and prescription. Baltimore (MD): Lippincott Williams \& Wilkins; 2000.

18. Rossier P, Wade DT. Validity and reliability comparison of 4 mobility measures in patients presenting with neurologic impairment. Arch Phys Med Rehabil. 2001;82(1):9-13. [PMID: 11239279]

19. Enright PL. The six-minute walk test. Respir Care. 2003; 48(8):783-85. [PMID: 12890299] 
JRRD, Volume 45, Number 2, 2008

20. Macko RF, Katzel LI, Yataco A, Tretter LD, DeSouza CA, Dengel DR, Smith GV, Silver KH. Low-velocity graded treadmill stress testing in hemiparetic stroke patients. Stroke. 1997;28(5):988-92. [PMID: 9158639]

21. Macko RF, DeSouza CA, Tretter LD, Silver KH, Smith GV, Anderson PA, Tomoyasu N, Gorman P, Dengel DR. Treadmill aerobic exercise training reduces the energy expenditure and cardiovascular demands of hemiparetic gait in chronic stroke patients. A preliminary report. Stroke. 1997; 28(2):326-30. [PMID: 9040684]

22. Michael KM, Allen JK, Macko RF. Reduced ambulatory activity after stroke: The role of balance, gait, and cardiovascular fitness. Arch Phys Med Rehabil. 2005;86(8):1552-56. |[PMID: 16084807]

23. Hsu AL, Tang PF, Jan MH. Analysis of impairments influencing gait velocity and asymmetry of hemiplegic patients after mild to moderate stroke. Arch Phys Med Rehabil. 2003; 84(8):1185-93. [PMID: 12917858]

24. Titianova EB, Pitkänen K, Pääkkönen A, Sivenius J, Tarkka IM. Gait characteristics and functional ambulation profile in patients with chronic unilateral stroke. Am J Phys Med Rehabil. 2003;82(10):778-86. [PMID: 14508410]

25. Balasubramanian CK, Bowden MG, Neptune RR, Kautz SA. Relationship between step length asymmetry and walk- ing performance in subjects with chronic hemiparesis. Arch Phys Med Rehabil. 2007;88(1):43-49. [PMID: 17207674$]$

26. Winter DA, Patla AE, Frank JS, Walt SE. Biomechanical walking pattern changes in the fit and healthy elderly. Phys Ther. 1990;70(6):340-47. [PMID: 2345777]

27. Guralnik JM, Ferrucci L, Pieper CF, Leveille SG, Markides KS, Ostir GV, Studenski S, Berkman LF, Wallace RB. Lower extremity function and subsequent disability: Consistency across studies, predictive models, and value of gait speed alone compared with the short physical performance battery. J Gerontol A Biol Sci Med Sci. 2000;55(4):M221-31. [PMID: 10811152]

28. Maki B. Gait changes in older adults: Predictors of falls or indicators of fear. J Am Geriatr Soc. 1997;45(3):313-20. [PMID: 9063277]

29. Luft AR, Macko R, Forrester L, Villagra F, Hanley D. Subcortical reorganization induced by aerobic locomotor training in chronic stroke survivors. Program No. 865.8. In: 2005 Abstract Viewer/Itinerary Planner, Society for Neuroscience 2005 Annual Meeting; 2005 Nov 12-16; Washington, DC. Washington (DC): Society for Neuroscience; 2005.

Submitted for publication February 2, 2007. Accepted in revised form September 5, 2007. 